\title{
Venous ulcer review
}

This article was published in the following Dove Press journal:

Clinical, Cosmetic and Investigational Dermatology

3 March 20II

Number of times this article has been viewed

\section{Paul Bevis \\ Jonothan Earnshaw}

Department of Vascular Surgery, Gloucestershire Royal Hospital, Great Western Road, Gloucester, UK

Date of preparation: 3 February 20II

Conflict of interest: None declared.
Correspondence: Jonothan Earnshaw Department of Vascular Surgery,

Gloucestershire Royal Hospital, Great Western Road, Gloucester, GLI 3NN, UK

$\mathrm{Tel}+448454226190$

Fax +44 (0) 8454226788

Email jjearnshaw@tiscali.co.uk
Clinical question: What is the best treatment for venous ulcers?

Results: Compression aids ulcer healing. Pentoxifylline can aid ulcer healing. Artificial skin grafts are more effective than other skin grafts in helping ulcer healing. Correction of underlying venous incompetence reduces ulcer recurrence.

Implementation: Potential pitfalls to avoid are:

- Failure to exclude underlying arterial disease before application of compression.

- Unusual-looking ulcers or those slow to heal should be biopsied to exclude malignant transformation.

Keywords: venous ulceration, ulcer healing

\section{Venous ulceration}

Definition: A skin defect in a limb with a venous abnormality.

Incidence: A $0.15 \%$ point prevalence with women outnumbering men $2.8: 1{ }^{1}$

Economics: An unhealed leg ulcer costs approximately $£ 1300$ per year to treat. ${ }^{2}$ Levels of evidence used in this summary: Systematic reviews, meta-analyses, and randomized controlled trials.

Search sources: PubMed, Cochrane Library, clinical evidence, and Google Scholar.

Outcomes: Ulcer healing, time to ulcer healing, pain relief during treatment, and prevention of ulcer recurrence.

Consumer summary: A venous ulcer is a complication of varicose veins. Venous ulcers can be slow to heal and impact on patients' quality of life. There is good evidence that compression helps heal ulcers. In patients who do not tolerate continuous compression, intermittent compression may help healing. In slow-healing ulcers, the use of pentoxifylline and bilayer artificial skin in conjunction with compression may aid healing. Surgery to incompetent veins reduces the risk of recurrence and endovenous surgery can speed ulcer healing.

\section{The evidence}

\section{Does compression aid ulcer healing?}

The following were analyzed:

Systematic reviews: 2

Meta-analysis: $\quad 0$

Randomized controlled trials: $\quad 26$ 
One systematic review ${ }^{3}$ concluded that 'compression increases ulcer healing rates compared with no compression. Multicomponent systems are more effective than single component systems. Multicomponent systems containing an elastic bandage appear more effective than those composed mainly of inelastic constituents'.

The second systematic review ${ }^{4}$ concluded that '... patients with venous leg ulcers treated with four-layer bandages experience faster healing than those treated with short-stretch bandages'.

The randomized trials show a benefit of compression over no compression. They also tend to favor multilayer, long-stretch compression over short-stretch compression (Table 1).

\section{Conclusions}

Compression aids ulcer healing.

\section{Does intermittent pneumatic compression aid ulcer healing?}

The following were analyzed:

Systematic reviews: 1

Meta-analysis: 0

Randomized controlled trials: $\quad 5$

The systematic review ${ }^{32}$ concluded that 'IPC may increase healing compared to no compression, but it is not clear whether it increases healing when added to treatment with bandages or if it can be used instead of compression bandages'.

\section{Randomized trials}

Two trials have shown a benefit for intermittent pneumatic compression (IPC) with a benefit for fast IPC over slow IPC in one trial. The other two trials didn't show a benefit for IPC (Table 2).

\section{Conclusions}

IPC may help healing when continuous compression cannot be tolerated.

\section{Does pentoxifylline aid the healing of venous ulcers?}

The following were analyzed:

Systematic reviews: 1

Meta-analysis: $\quad 0$

Randomized controlled trials: 6

The systematic review concluded that 'pentoxifylline is an effective adjunct to compression bandaging for treating venous ulcers and may be effective in the absence of compression'. 39

\section{Randomized trials}

All trials showed increased healing in the pentoxifylline group with no benefit shown for higher doses (Table 3).

\section{Conclusions}

Pentoxifylline $400 \mathrm{mg}$ tds has a role in aiding the healing of venous ulcers.

\section{Does skin grafting aid ulcer healing?}

The following were analyzed:

Systematic reviews: $\quad 1$

Meta-analysis: 0

Randomized controlled trials: $\quad 11$

The systematic review ${ }^{46}$ concluded that 'bilayer artificial skin, used in conjunction with compression bandaging, increases venous ulcer healing compared with a simple dressing plus compression. Further research is needed to assess whether other forms of skin grafts increase ulcer healing'.

\section{Randomized trials}

Increased healing was seen compared to no grafting with the greatest difference seen with artificial skin grafts (Table 4).

\section{Conclusions}

Artificial skin helps a greater proportion of ulcers heal than other skin grafts.

\section{Does surgery or endovenous therapy aid ulcer healing and prevent recurrence?}

The following were analyzed:

Systematic review: 1

Meta-analysis: 0

Randomized controlled trials: $\quad 5$

The systematic review ${ }^{58}$ concluded that ' ... superficial venous surgery is associated with similar rates of ulcer healing to compression alone, but with less recurrence'.

\section{Randomized trials}

Only endovenous surgery seems to aid ulcer healing, but all forms of surgery reduce ulcer recurrence (Table 5).

\section{Conclusions}

Correction of venous incompetence is important to reduce the incidence of ulcer recurrence after healing. 
Table I Randomized controlled trials showing the effect of compression on ulcer healing

\begin{tabular}{|c|c|c|c|c|}
\hline Author & $\begin{array}{l}\text { Number } \\
\text { randomized }\end{array}$ & Interventions & Outcome measures & Results \\
\hline $\begin{array}{l}\text { Hendricks } \\
\text { and Swallow }\end{array}$ & 21 & $\begin{array}{l}\text { GpI: Unna's boot } \\
\text { Gp2: below-knee elastic compression } \\
\text { stocking }\end{array}$ & Healing at 78 weeks & $\begin{array}{l}\text { Gpl: } 70 \% \text { healed } \\
\text { Gp2: } 71 \% \text { healed }\end{array}$ \\
\hline Eriksson $^{6}$ & 34 & $\begin{array}{l}\text { GpI: inner stocking plus outer } \\
\text { elastic bandage Gp2: hydrocolloid } \\
\text { dressing plus elastic bandage }\end{array}$ & Healing at 12 weeks & $\begin{array}{l}\text { GpI: } 41 \% \text { healed } \\
\text { Gp2: } 53 \% \text { healed }\end{array}$ \\
\hline Kikta et $\mathrm{al}^{7}$ & 87 & $\begin{array}{l}\text { Gpl: Unna's boot } \\
\text { Gp2: no compression }\end{array}$ & Healing at 6 months & $\begin{array}{l}\text { Gpl: } 70 \% \text { healed } \\
\text { Gp2: } 38 \% \text { healed }\end{array}$ \\
\hline Rubin et $\mathrm{al}^{8}$ & 36 & $\begin{array}{l}\text { GpI: Unna's boot } \\
\text { Gp2: polyurethane foam dressing }\end{array}$ & Healing at 12 months & $\begin{array}{l}\text { GpI: } 95 \% \text { healed } \\
\text { Gp2: } 41 \% \text { healed }\end{array}$ \\
\hline Charles $^{9}$ & 53 & $\begin{array}{l}\text { Gpl: short-stretch compression } \\
\text { Gp2: usual care (no compression) }\end{array}$ & Healing at 3 months & $\begin{array}{l}\text { GpI: } 71 \% \text { healed } \\
\text { Gp2: } 25 \% \text { healed }\end{array}$ \\
\hline Cordts et al ${ }^{10}$ & 43 & $\begin{array}{l}\text { Gpl: hydrocolloid dressing plus } \\
\text { cohesive elastic bandage } \\
\text { Gp2: Unna's boot }\end{array}$ & Healing at 12 weeks & $\begin{array}{l}\text { GpI: } 50 \% \text { healed } \\
\text { Gp2: } 43 \% \text { healed }\end{array}$ \\
\hline Travers et al' ${ }^{\prime \prime}$ & 27 & $\begin{array}{l}\text { GpI: single-layer elastic cohesive } \\
\text { bandage Gp2: 3-layer compression }\end{array}$ & $\begin{array}{l}\text { Mean percentage } \\
\text { change at } 7 \text { weeks }\end{array}$ & Gpl: $-90 \%$ Gp2: $-83 \%$ \\
\hline Danielsen et al ${ }^{12}$ & 43 & $\begin{array}{l}\text { Gpl: long-stretch, nonadhesive } \\
\text { compression bandage Gp2: } \\
\text { short-stretch, nonadhesive } \\
\text { compression bandage }\end{array}$ & $\begin{array}{l}\text { Healing at } 6 \text { and } \\
12 \text { months }\end{array}$ & $\begin{array}{l}\text { GpI: } 39 \% \text { healed at } 6 \text { months } \\
\text { and } 52 \% \text { at } 12 \text { months } \\
\text { Gp2: } 25 \% \text { healed at } 6 \text { months } \\
\text { and } 15 \% \text { at } 12 \text { months }\end{array}$ \\
\hline Gould et $\mathrm{al}^{13}$ & 46 & $\begin{array}{l}\text { GpI: 3-component, long-stretch } \\
\text { compression Gp2: 3-component, } \\
\text { short-stretch compression }\end{array}$ & Healing at 15 weeks & $\begin{array}{l}\text { GpI: } 58 \% \text { healed } \\
\text { Gp2: } 35 \% \text { healed }\end{array}$ \\
\hline Morrell et al $\left.\right|^{14,15}$ & 233 & $\begin{array}{l}\text { Gpl: 4-layer compression } \\
\text { Gp2: standard community care }\end{array}$ & Healing at 12 months & $\begin{array}{l}\text { GpI: } 65 \% \text { healed } \\
\text { Gp2: } 55 \% \text { healed }\end{array}$ \\
\hline Scriven et $\mathrm{al}^{16}$ & 64 & $\begin{array}{l}\text { Gp1: 4-layer compression } \\
\text { Gp2: short-stretch compression }\end{array}$ & Healing at 12 months & $\begin{array}{l}\text { GpI: } 55 \% \text { healed } \\
\text { Gp2: } 57 \% \text { healed }\end{array}$ \\
\hline Taylor et al ${ }^{17}$ & 36 & $\begin{array}{l}\text { Gpl: 4-layer compression } \\
\text { Gp2: standard community care }\end{array}$ & Healing at 12 weeks & $\begin{array}{l}\text { GpI: } 67 \% \text { healed } \\
\text { Gp2: } 17 \% \text { healed }\end{array}$ \\
\hline Moody ${ }^{18}$ & 52 & $\begin{array}{l}\text { Gpl: short-stretch compression } \\
\text { Gp2: long-stretch compression }\end{array}$ & Healing at 12 weeks & $\begin{array}{l}\text { GpI: } 31 \% \text { healed } \\
\text { Gp2: } 31 \% \text { healed }\end{array}$ \\
\hline Vowden et al ${ }^{19}$ & 149 & $\begin{array}{l}\text { Gpl: Charing Cross 4-layer } \\
\text { compression Gp2: modified } \\
\text { 4-layer compression Gp3: 4-layer } \\
\text { compression bandage kit }\end{array}$ & Healing at 12 weeks & $\begin{array}{l}\text { GpI: } 60 \% \text { healed } \\
\text { Gp2: } 76 \% \text { healed } \\
\text { Gp3: } 60 \% \text { healed }\end{array}$ \\
\hline Partsch et $\mathrm{a}^{20}$ & 112 & $\begin{array}{l}\text { Gpl: 4-layer compression } \\
\text { Gp2: short-stretch compression }\end{array}$ & Healing at 16 weeks & $\begin{array}{l}\text { Gpl: } 62 \% \text { healed } \\
\text { Gp2: } 73 \% \text { healed }\end{array}$ \\
\hline Moffatt et al ${ }^{21}$ & 112 & $\begin{array}{l}\text { Gpl: 4-layer compression } \\
\text { Gp2: 2-layer compression }\end{array}$ & Healing at 12 weeks & $\begin{array}{l}\text { GpI: } 70 \% \text { healed } \\
\text { Gp2: } 58 \% \text { healed }\end{array}$ \\
\hline O'Brien et $\mathrm{al}^{22}$ & 200 & $\begin{array}{l}\text { Gpl: 4-layer compression } \\
\text { Gp2: standard community care }\end{array}$ & Healing at 12 weeks & $\begin{array}{l}\text { GpI: } 54 \% \text { healed } \\
\text { Gp2: } 34 \% \text { healed }\end{array}$ \\
\hline Ukat et $\mathrm{al}^{23}$ & 89 & $\begin{array}{l}\text { Gp1: 4-layer compression } \\
\text { Gp2: short-stretch compression }\end{array}$ & Healing at 12 weeks & $\begin{array}{l}\text { Gpl: } 30 \% \text { healed } \\
\text { Gp2: } 22 \% \text { healed }\end{array}$ \\
\hline Franks et $\mathrm{a}^{24}$ & 159 & $\begin{array}{l}\text { Gp1: 4-layer compression } \\
\text { Gp2: short-stretch compression }\end{array}$ & Healing at 24 weeks & $\begin{array}{l}\text { Gpl: } 69 \% \text { healed } \\
\text { Gp2: } 73 \% \text { healed }\end{array}$ \\
\hline Nelson et $\mathrm{a}^{25}$ & 387 & $\begin{array}{l}\text { Gpl: 4-layer compression } \\
\text { Gp2: short-stretch bandage }\end{array}$ & $\begin{array}{l}\text { Healing at } 4 \text { and } \\
12 \text { months }\end{array}$ & $\begin{array}{l}\text { GpI: } 55 \% \text { healed at } 4 \text { months } \\
\text { and } 78 \% \text { healed at } 12 \text { months } \\
\text { Gp2: } 45 \% \text { healed at } 4 \text { months } \\
\text { and } 72 \% \text { at } 12 \text { months }\end{array}$ \\
\hline Jünger et $\mathrm{a}^{26}$ & 134 & $\begin{array}{l}\text { Gpl: U-stocking consisting } \\
\text { of two stockings } \\
\text { Gp2: short-stretch bandages }\end{array}$ & Healing at 12 weeks & $\begin{array}{l}\text { GpI: } 48 \% \text { healed } \\
\text { Gp2: } 32 \% \text { healed }\end{array}$ \\
\hline
\end{tabular}


Table I (Continued)

\begin{tabular}{|c|c|c|c|c|}
\hline Author & $\begin{array}{l}\text { Number } \\
\text { randomized }\end{array}$ & Interventions & Outcome measures & Results \\
\hline \multirow[t]{2}{*}{ Nelson $^{27}$} & 133 & Gpl: 3-layer compression & Healing at 52 weeks & Gpl: $80 \%$ healed \\
\hline & & Gp2: 4-layer compression & & Gp2: $65 \%$ healed \\
\hline \multirow[t]{2}{*}{ Polignano et $\mathrm{al}^{28}$} & 68 & GpI: 4-layer compression & Healing at 24 weeks & Gpl: 74\% healed \\
\hline & & Gp2: Unna's boot & & Gp2: $66 \%$ healed \\
\hline \multirow[t]{2}{*}{ Polignano et $\mathrm{al}^{29}$} & 56 & Gpl: short-stretch compression & Healing at 12 weeks & Gpl: I7\% healed \\
\hline & & $\begin{array}{l}\text { Gp2: multilayer high compression } \\
\text { system }\end{array}$ & & Gp2: $44 \%$ healed \\
\hline \multirow[t]{2}{*}{ Blecken et $\mathrm{al}^{30}$} & 12 & Gpl: adjustable compression boot & Healing at 12 weeks & Gpl: $93 \%$ healed \\
\hline & & system Gp2: 4-layer compression & & Gp2: $51 \%$ healed \\
\hline \multirow[t]{2}{*}{ Milic et $\mathrm{al}^{31}$} & 150 & Gpl: tubular compression device & Healing at 500 days & Gpl: $33 \%$ healed \\
\hline & & $\begin{array}{l}(35-40 \mathrm{~mm} \mathrm{Hg}) \mathrm{Gp} 2: 2 \text { medium- } \\
\text { stretch compression bandages } \\
(20-25 \mathrm{~mm} \mathrm{Hg})\end{array}$ & & Gp2: 33\% healed \\
\hline
\end{tabular}

Abbreviations: Gpl, group 1; Gp2, group 2; Gp3, group 3.

\section{The practice}

\section{Potential pitfalls}

There is a small rate of malignant transformation in ulcers (4.4\%), $75 \%$ basal cell carcinoma, and $25 \%$ squamous cell carcinoma. ${ }^{64}$ Ulcers in unusual locations, with irregular edges, those with islands of epithelium that do not persist, or those slow to heal should be biopsied. ${ }^{64}$

\section{Management}

Venous leg ulceration can often be managed in the community or in nurse-led venous ulcer clinics. Indications for specialist referral are detailed below.

\section{Assessment}

Nutritional status of patients should be assessed.

- There may be a history of varicose veins.

- Any history of intravenous injection should be elicited.

- Any medication or medical condition potentially affecting healing should be assessed.

- Concomitant arterial disease should be excluded using ankle brachial pressure indices before the application of any compression.

- Patients should be examined for evidence of superficial venous incompetence.

- Any history of deep vein thrombosis should be elicited.

Table 2 Randomized controlled trials showing the effects of intermittent pneumatic compression on ulcer healing

\begin{tabular}{|c|c|c|c|c|}
\hline Author & $\begin{array}{l}\text { Number } \\
\text { randomized }\end{array}$ & Interventions & $\begin{array}{l}\text { Outcome } \\
\text { measures }\end{array}$ & Results \\
\hline Smith et $\mathrm{a}^{33}$ & 45 & $\begin{array}{l}\text { Both groups had same dressings } \\
\text { and stockings. Sequential IPC for } \\
\text { up to } 4 \mathrm{~h} \text { in one group }\end{array}$ & Healing & $\begin{array}{l}48 \% \text { healed in IPC group } \\
\text { and } 4 \% \text { in control group }\end{array}$ \\
\hline McCulloch et a ${ }^{34}$ & 22 & $\begin{array}{l}\text { Both groups had the same dressings } \\
\text { and Unna's boots. IPC for } 60 \mathrm{~min} \\
\text { twice weekly in one group }\end{array}$ & Healing & $\begin{array}{l}100 \% \text { healed in IPC group } \\
\text { and } 80 \% \text { in control group }\end{array}$ \\
\hline Schuler et $\mathrm{a}^{35}$ & 53 & $\begin{array}{l}\text { Unna's boots versus elasticated } \\
\text { stockings plus IPC for } 60 \mathrm{~min} \text { in the } \\
\text { morning and } 120 \mathrm{~min} \text { in the evening }\end{array}$ & Healing & $\begin{array}{l}71 \% \text { healed in IPC group } \\
\text { and } 75 \% \text { in Unna's boot group }\end{array}$ \\
\hline Rowland $^{36}$ & 16 & $\begin{array}{l}\text { Crossover trial of dressing alone } \\
\text { with dressing and IPC for } 60 \mathrm{~min} \\
\text { twice daily for } 2-3 \text { months }\end{array}$ & Healing & $\begin{array}{l}\text { No ulcers healed in either } \\
\text { arm before crossover }\end{array}$ \\
\hline Kumar et al ${ }^{37}$ & 47 & $\begin{array}{l}\text { Both groups had 4-layer bandaging } \\
\text { IPC for } 60 \text { min twice daily for } 4 \text { months in } \\
\text { one group }\end{array}$ & Healing & $\begin{array}{l}87 \% \text { healed in IPC group } \\
\text { and } 92 \% \text { in control group }\end{array}$ \\
\hline Nikolovska et a $\left.\right|^{38}$ & 104 & $\begin{array}{l}\text { Both groups had same dressings } \\
\text { Fast IPC for one group and slow } \\
\text { IPC in the other group }\end{array}$ & $\begin{array}{l}\text { Healing at } \\
6 \text { months }\end{array}$ & $\begin{array}{l}86 \% \text { healed with fast IPC } \\
\text { and } 61 \% \text { with slow IPC }\end{array}$ \\
\hline
\end{tabular}

Abbreviation: IPC, intermittent pneumatic compression. 
Table 3 Randomized controlled trials showing the effect of pentoxifylline on ulcer healing

\begin{tabular}{|c|c|c|c|c|}
\hline Author & $\begin{array}{l}\text { Number } \\
\text { randomized }\end{array}$ & Interventions & $\begin{array}{l}\text { Outcome } \\
\text { measures }\end{array}$ & Results \\
\hline Colgan et $\mathrm{al}^{40}$ & 80 & $\begin{array}{l}\text { All had 2-layer compression } \\
\text { Gpl: } 400 \text { mg tds pentoxifylline } \\
\text { Gp2: placebo }\end{array}$ & $\begin{array}{l}\text { Healing at } 24 \\
\text { weeks }\end{array}$ & $\begin{array}{l}\text { Gpl: } 60 \% \text { healed } \\
\text { Gp2: } 29 \% \text { healed }\end{array}$ \\
\hline Barbarino $^{4 l}$ & 12 & $\begin{array}{l}\text { All had 2-layer compression } \\
\text { Gpl: } 400 \text { mg tds pentoxifylline } \\
\text { Gp2: placebo }\end{array}$ & Healing & $\begin{array}{l}\text { Gpl: } 66 \% \text { healed } \\
\text { Gp2: } 17 \% \text { healed }\end{array}$ \\
\hline Dale et $\mathrm{al}^{42}$ & 200 & $\begin{array}{l}\text { All had compression } \\
\text { Gpl:400 mg tds pentoxifylline } \\
\text { Gp2: placebo }\end{array}$ & $\begin{array}{l}\text { Healing at } 24 \\
\text { weeks }\end{array}$ & $\begin{array}{l}\text { Gpl: } 64 \% \text { healed } \\
\text { Gp2: } 52 \% \text { healed }\end{array}$ \\
\hline Falanga et $\mathrm{al}^{43}$ & 129 & $\begin{array}{l}\text { All had compression } \\
\text { Gpl: } 800 \text { mg tds pentoxifylline } \\
\text { Gp2: } 400 \text { mg tds pentoxifylline } \\
\text { Gp3: placebo }\end{array}$ & $\begin{array}{l}\text { Healing at } 24 \\
\text { weeks }\end{array}$ & $\begin{array}{l}\text { Gpl: } 73 \% \text { healed } \\
\text { Gp2: } 75 \% \text { healed } \\
\text { Gp3: } 63 \% \text { healed }\end{array}$ \\
\hline Belcaro et $\mathrm{al}^{44}$ & 172 & $\begin{array}{l}\text { All had 2-layer compression } \\
\text { Gpl: } 400 \text { mg tds pentoxifylline } \\
\text { Gp2: placebo }\end{array}$ & $\begin{array}{l}\text { Healing and } \\
\text { reduction in } \\
\text { ulcer size }\end{array}$ & $\begin{array}{l}\text { Gpl: } 65 \% \text { healed, } \\
87 \% \text { size reduction } \\
\text { Gp2: } 27 \% \text { healed, } \\
47 \% \text { size reduction }\end{array}$ \\
\hline Nikolovska et $\mathrm{al}^{45}$ & 80 & $\begin{array}{l}\text { All had hydrocolloid dressing } \\
\text { One group had } 400 \mathrm{mg} \text { tds } \\
\text { pentoxifylline }\end{array}$ & $\begin{array}{l}\text { Healing at } 24 \\
\text { weeks }\end{array}$ & $\begin{array}{l}58 \% \text { healed in } \\
\text { pentoxifylline group } \\
\text { and } 28 \% \text { in no tablet } \\
\text { group }\end{array}$ \\
\hline
\end{tabular}

Abbreviations: Gpl, group I; Gp2, group 2; Gp3, group 3.

Table 4 Randomized controlled trials showing the effect of different types of skin grafting on ulcer healing

\begin{tabular}{|c|c|c|c|c|}
\hline Author & $\begin{array}{l}\text { Number } \\
\text { randomized }\end{array}$ & Interventions & Outcome measures & Results \\
\hline Poskitt et $\mathrm{al}^{47}$ & 53 & $\begin{array}{l}\text { Both groups received } \\
\text { compression } \\
\text { Gpl: pinch skin grafts } \\
\text { Gp2: porcine dermis }\end{array}$ & Healing at 6 and 12 weeks & $\begin{array}{l}\text { Gpl: } 64 \% \text { healed at } 6 \text { weeks } \\
\text { and } 72 \% \text { at } 12 \text { weeks } \\
\text { Gp2: } 29 \% \text { healed at } 6 \text { weeks } \\
\text { and } 46 \% \text { healed at } 12 \text { weeks }\end{array}$ \\
\hline Mol et $\mathrm{al}^{48}$ & II & $\begin{array}{l}\text { Gpl: human skin equivalents } \\
\text { Gp2: punch grafts }\end{array}$ & Healing at 20 days & $\begin{array}{l}\text { Gpl: } 80 \% \text { healed } \\
\text { Gp2: 71\% healed }\end{array}$ \\
\hline Teepe et $\mathrm{al}^{49}$ & 47 & $\begin{array}{l}\text { Both groups received short- } \\
\text { stretch bandages } \\
\text { Gpl: cryopreserved allografts } \\
\text { Gp2: controls }\end{array}$ & Healing at 6 weeks & $\begin{array}{l}\text { Gpl: } 25 \% \text { healed } \\
\text { Gp2: } 22 \% \text { healed }\end{array}$ \\
\hline Warburg et $\mathrm{al}^{50}$ & 31 & $\begin{array}{l}\text { Both groups received } \\
\text { compression Gpl: meshed } \\
\text { split-skin graft Gp2: surgery } \\
\text { for incompetent perforators }\end{array}$ & Healing at 12 months & $\begin{array}{l}\text { Gpl: } 33 \% \text { healed } \\
\text { Gp2: } 38 \% \text { healed }\end{array}$ \\
\hline Falanga et $\mathrm{al}^{51}$ & 309 & $\begin{array}{l}\text { All received compression } \\
\text { Gpl: human skin equivalent } \\
\text { Gp2: dressing }\end{array}$ & Healing at 6 months & $\begin{array}{l}\text { Gpl: } 63 \% \text { healed } \\
\text { Gp2: } 49 \% \text { healed }\end{array}$ \\
\hline Lindgren et $\mathrm{al}^{52}$ & 27 & $\begin{array}{l}\text { Both groups received } \\
\text { compression } \\
\text { Gpl: cryopreserved allografts } \\
\text { Gp2: no graft }\end{array}$ & Healing at 8 weeks & $\begin{array}{l}\text { Gpl: } 13 \% \text { healed } \\
\text { Gp2: } 17 \% \text { healed }\end{array}$ \\
\hline Tausche et $\mathrm{al}^{53}$ & 92 & $\begin{array}{l}\text { Gpl: autologous epidermal } \\
\text { equivalents derived from hair } \\
\text { follicles Gp2: meshed skin } \\
\text { autograft }\end{array}$ & Healing at 6 months & $\begin{array}{l}\text { Gpl: } 42 \% \text { healed } \\
\text { Gp2: } 34 \% \text { healed }\end{array}$ \\
\hline
\end{tabular}


Table 4 (Continued)

\begin{tabular}{|c|c|c|c|c|}
\hline Author & $\begin{array}{l}\text { Number } \\
\text { randomized }\end{array}$ & Interventions & Outcome measures & Results \\
\hline Krishnamoorthy et a ${ }^{54}$ & 53 & $\begin{array}{l}\text { All received 4-layer } \\
\text { compression } \\
\text { GpI: Dermagraft, weekly for } \\
\text { I2 applications } \\
\text { Gp2: Dermagraft at } 0,1,4 \text {, } \\
\text { and } 8 \text { weeks } \\
\text { Gp3: Dermagraft at } 0 \text { weeks } \\
\text { Gp4: No Dermagraft }\end{array}$ & Healing at 12 weeks & $\begin{array}{l}\text { GpI: } 38 \% \text { healed } \\
\text { Gp2: } 38 \% \text { healed } \\
\text { Gp3: } 7 \% \text { healed } \\
\text { Gp4: } 15 \% \text { healed }\end{array}$ \\
\hline Liu et $\mathrm{a}^{55}$ & 10 & $\begin{array}{l}\text { Both groups had ulcers } \\
\text { debrided and multilayer } \\
\text { compression bandaging } \\
\text { GpI: keratinocytes cultured } \\
\text { on porcine gelatin microbeads } \\
\text { Gp2: keratinocytes cultured } \\
\text { on porcine collagen pads }\end{array}$ & $\begin{array}{l}\text { Healing at } 12 \text { weeks } \\
\mathrm{s}\end{array}$ & $25 \%$ healed in both groups \\
\hline Navrátilová et al ${ }^{56}$ & 50 & $\begin{array}{l}\text { Gpl: cryopreserved cultured } \\
\text { epidermal keratinocytes } \\
\text { Gp2: lyophilized cultured } \\
\text { epidermal keratinocytes }\end{array}$ & Healing at 90 days & $\begin{array}{l}\text { GpI: } 84 \% \text { healed } \\
\text { Gp2: } 80 \% \text { healed }\end{array}$ \\
\hline Omar et $\mathrm{a}^{57}$ & 18 & $\begin{array}{l}\text { Both groups received 4-layer } \\
\text { bandaging Gp I: Dermagraft } \\
\text { Gp2: no graft }\end{array}$ & Healing at 12 weeks & $\begin{array}{l}\text { GpI: } 50 \% \text { healed } \\
\text { Gp2: I } 3 \% \text { healed }\end{array}$ \\
\hline
\end{tabular}

Abbreviations: Gpl, group I; Gp2, group 2; Gp3, group 3; Gp4, group 4.

Table 5 Randomized controlled trials showing the effect of different types of surgery and endovenous therapy on ulcer healing and recurrence

\begin{tabular}{|c|c|c|c|c|}
\hline Author & $\begin{array}{l}\text { Number } \\
\text { randomized }\end{array}$ & Interventions & $\begin{array}{l}\text { Outcome } \\
\text { measures }\end{array}$ & Results \\
\hline Guest et $\mathrm{al}^{59}$ & 76 & $\begin{array}{l}\text { Gpl: compression alone } \\
\text { Gp2: compression and } \\
\text { superficial venous surgery } \\
\pm \text { perforator surgery }\end{array}$ & Healing & $\begin{array}{l}\text { GpI: } 64 \% \text { healed } \\
\text { Gp2: } 68 \% \text { healed }\end{array}$ \\
\hline Zamboni et $a^{60}$ & 45 & $\begin{array}{l}\text { GpI: compression alone } \\
\text { Gp2: compression and } \\
\text { minimally invasive surgical } \\
\text { hemodynamic correction of } \\
\text { reflux }\end{array}$ & $\begin{array}{l}\text { Healing and } \\
\text { recurrence }\end{array}$ & $\begin{array}{l}\text { GpI: } 96 \% \text { healed, } \\
38 \% \text { recurrence } \\
\text { Gp2: } 100 \% \text { healed, } \\
9 \% \text { recurrence }\end{array}$ \\
\hline Van Gent et $\mathrm{a}^{61}$ & 200 & $\begin{array}{l}\text { Gpl: compression alone } \\
\text { Gp2: compression and } \\
\text { subfascial endoscopic } \\
\text { perforating vein surgery }\end{array}$ & $\begin{array}{l}\text { Healing and } \\
\text { recurrence }\end{array}$ & $\begin{array}{l}\text { GpI: } 73 \% \text { healed, } \\
23 \% \text { recurrence } \\
\text { Gp2: } 83 \% \text { healed, } \\
22 \% \text { recurrence }\end{array}$ \\
\hline Gohel et a ${ }^{62}$ & 500 & $\begin{array}{l}\text { Gpl: compression alone } \\
\text { Gp2: compression and } \\
\text { superficial venous surgery }\end{array}$ & $\begin{array}{l}\text { Ulcer healing } \\
\text { and ulcer } \\
\text { recurrence at } \\
3 \text { years }\end{array}$ & $\begin{array}{l}\text { GpI: } 89 \% \text { healed, } \\
56 \% \text { recurrence } \\
\text { Gp2: } 93 \% \text { healed, } \\
31 \% \text { recurrence }\end{array}$ \\
\hline Viarengo et $\mathrm{a}^{63}$ & 52 & $\begin{array}{l}\text { Gpl: compression alone } \\
\text { Gp2: endovenous laser } \\
\text { therapy and compression }\end{array}$ & $\begin{array}{l}\text { Healing at } \\
12 \text { months }\end{array}$ & $\begin{array}{l}\text { GpI: } 24 \% \text { healed } \\
\text { Gp2: } 82 \% \text { healed }\end{array}$ \\
\hline
\end{tabular}

Abbreviations: GpI, group I; Gp2, group 2. 


\section{Treatment}

A 4-layer compression, if tolerated.

- Short-stretch compression or intermittent compression if 4-layer not tolerated.

- Pentoxifylline (400 mg three times daily) and skin grafting should be considered if ulcers are slow to heal.

- Incompetent veins should be treated to reduce the risk of ulcer recurrence.

\section{Indications for specialist referral}

Worsening despite treatment or slow healing.

- Unusual appearance of ulcer.

\section{References}

1. Callam MJ, Ruckley CV, Harper DR, Dale JJ. Chronic ulceration of the leg: extent of the problem and provision of care. Br Med J (Clin Res Ed). 1985;290(6485):1855-1856.

2. Iglesias CP, Nelson EA, Cullum N, Torgerson DJ; VenUS I collaborators. Economic analysis of VenUS I, a randomized trial of two bandages for treating venous leg ulcers. Br J Surg. 2004;91(10):1300-1306.

3. O'Meara S, Cullum NA, Nelson EA. Compression for venous leg ulcers. Cochrane Database Syst Rev. 2009;(1):CD000265.

4. O'Meara S, Tierney J, Cullum N, et al. Four layer bandage compared with short stretch bandage for venous leg ulcers: systematic review and meta-analysis of randomised controlled trials with data from individual patients. BMJ. 2009;338:b1344.

5. Hendricks WM, Swallow RT. Management of stasis leg ulcers with Unna's boots versus elastic support stockings. J Am Acad Dermatol. 1985;12(1 Pt 1):90-98.

6. Eriksson G. Comparison of two occlusive bandages in the treatment of venous leg ulcers. Br J Dermatol. 1986;114(2):227-230.

7. Kikta MJ, Schuler JJ, Meyer JP, et al. A prospective, randomized trial of Unna's boots versus hydroactive dressing in the treatment of venous stasis ulcers. J Vasc Surg. 1988;7(3):478-483.

8. Rubin JR, Alexander J, Plecha EJ, Marman C. Unna's boot vs polyurethane foam dressings for the treatment of venous ulceration. A randomized prospective study. Arch Surg. 1990;125(4):489-490.

9. Charles H. Compression healing of ulcers. J Dist Nurs. 1991;10(3):4-8.

10. Cordts PR, Hanrahan LM, Rodriguez AA, Woodson J, LaMorte WW, Menzoian JO. A prospective, randomized trial of Unna's boot versus Duoderm CGF hydroactive dressing plus compression in the management of venous leg ulcers. J Vasc Surg. 1992;15(3):480-486.

11. Travers JP, Dalziel KL, Makin GS. Assessment of a new one-layer adhesive bandaging method in maintaining prolonged limb compression and effects on venous ulcer healing. Phlebology. 1992;7:59-63.

12. Danielsen L, Madsen SM, Henriksen L. Venous leg ulcer healing. A randomised prospective study of long-stretch versus short-stretch compression bandages. Phlebology. 1998;13:59-63.

13. Gould DJ, Campbell S, Newton H, Duffelen P, Griffin M, Harding EF. Setopress vs Elastocrepe in chronic venous ulceration. Br J Nurs. 1998; 7(2):66-70, 72-73.

14. Morrell CJ, King B, Brereton L. Community-based leg ulcer clinics: organisation and cost-effectiveness. Nurs Times. 1998;94(9):51-54.

15. Morrell CJ, Walters SJ, Dixon S, et al. Cost effectiveness of com munity leg ulcer clinics: randomised controlled trial. $B M J$. 1998;316(7143):1487-1491.

16. Scriven JM, Taylor LE, Wood AJ, Bell PR, Naylor AR, London NJ. A prospective randomised trial of four-layer versus short stretch compression bandages for the treatment of venous leg ulcers. Ann R Coll Surg Engl. 1998;80(3):215-220.
17. Taylor AD, Taylor RJ, Marcuson RW. Prospective comparison of healing rates and therapy costs for conventional and four-layer high compression bandaging treatments of venous leg ulcers. Phlebology. 1998;13:20-24.

18. Moody M. Comparison of Rosidal K and SurePress in the treatment of venous leg ulcers. Br J Nurs. 1999;8(6):345-355.

19. Vowden KR, Mason A, Wilkinson D, Vowden P. Comparison of the healing rates and complications of three four-layer bandage regimens. J Wound Care. 2000;9(6):269-272.

20. Partsch H, Damstra RJ, Tazelaar DJ, et al. Multicentre, randomised controlled trial of four-layer bandaging versus short-stretch bandaging in the treatment of venous leg ulcers. Vasa. 2001;30(2): $108-113$.

21. Moffatt CJ, McCullagh L, O'Connor T, et al. Randomized trial of fourlayer and two-layer bandage systems in the management of chronic venous ulceration. Wound Repair Regen. 2003;11(3):166-171.

22. O'Brien JF, Grace PA, Perry IJ, Hannigan A, Clarke Moloney M, Burke PE. Randomized clinical trial and economic analysis of four-layer compression bandaging for venous ulcers. Br J Surg. 2003;90(7):794-798.

23. Ukat A, Konig M, Vanscheidt W, Münter KC. Short-stretch versus multilayer compression for venous leg ulcers: a comparison of healing rates. J Wound Care. 2003;12(4):139-143.

24. Franks PJ, Moody M, Moffatt CJ, et al; Wound Healing Nursing Research Group. Randomized trial of cohesive short-stretch versus four-layer bandaging in the management of venous ulceration. Wound Repair Regen. 2004;12(2):157-162.

25. Nelson EA, Iglesias CP, Cullum N, Torgerson DJ; VenUS I collaborators. Randomized clinical trial of four-layer and short-stretch compression bandages for venous leg ulcers (VenUS I). Br J Surg. 2004;91(10):1292-1299.

26. Jünger M, Wollina U, Kohnen R, Rabe E. Efficacy and tolerability of an ulcer compression stocking for therapy of chronic venous ulcer compared with a below-knee compression bandage: results from a prospective, randomized, multicentre trial. Curr Med Res Opin. 2004;20(10): 1613-1623.

27. Nelson EA. 3 layer paste bandages were more effective than 4 layer bandages for healing venous leg ulcers. Evid Based Nurs. 2004; $7(1): 21$.

28. Polignano R, Bonadeo P, Gasbarro S, Allegra C. A randomised controlled study of four-layer compression versus Unna's boot for venous ulcers. J Wound Care. 2004;13(1):21-24.

29. Polignano R, Guarnera G, Bonadeo P. Evaluation of SurePress Comfort: a new compression system for the management of venous leg ulcers. J Wound Care. 2004;13(9):387-391.

30. Blecken SR, Villavicencio JL, Kao TC. Comparison of elastic versus nonelastic compression in bilateral venous ulcers: a randomized trial. J Vasc Surg. 2005;42(6):1150-1155.

31. Milic DJ, Zivic SS, Bogdanovic DC, et al. A randomized trial of the Tubulcus multilayer bandaging system in the treatment of extensive venous ulcers. J Vasc Surg. 2007;46(4):750-755.

32. Nelson EA, Mani R, Vowden K. Intermittent pneumatic compression for treating venous leg ulcers. Cochrane Database Syst Rev. 2008;2:CD001899.

33. Smith PC, Sarin S, Hasty J, Scurr JH. Sequential gradient pneumatic compression enhances venous ulcer healing: a randomized trial. Surgery. 1990;108(5):871-875.

34. McCulloch JM, Marler KC, Neal MB, Phifer TJ. Intermittent pneumatic compression improves venous ulcer healing. Adv Wound Care. 1994;7(4):22-24, 26.

35. Schuler JJ, Maibenco T, Megerman J, Ware M, Montalvo J. Treatment of chronic venous ulcers using sequential gradient intermittent pneumatic compression. Phlebology. 1996;11(3):111-116.

36. Rowland J. Intermittent pump versus compression bandages in the treatment of venous leg ulcers. Aust N Z J Surg. 2000;70(2): $110-113$. 
37. Kumar S, Samraj K, Nirujogi V, Budnik J, Walker MA. Intermittent pneumatic compression as an adjuvant therapy in venous ulcer disease. J Tissue Viability. 2002;12(2):42-44, 46, 48 passim.

38. Nikolovska S, Arsovski A, Damevska K, Gocev G, Pavlova L. Evaluation of two different intermittent pneumatic compression cycle settings in the healing of venous ulcers: a randomized trial. Med Sci Monit. 2005;11(7):CR337-CR343.

39. Jull A, Arroll B, Parag V, Waters J. Pentoxifylline for treating venous leg ulcers. Cochrane Database Syst Rev. 2007;(3):CD001733.

40. Colgan MP, Dormandy JA, Jones PW, Schraibman IG, Shanik DG, Young RA. Oxpentifylline treatment of venous ulcers of the leg. $B M J$. 1990;300(6730):972-975.

41. Barbarino C. Pentoxifylline in the treatment of venous leg ulcers. Curr Med Res Opin. 1992;12(9):547-551.

42. Dale JJ, Ruckley CV, Harper DR, Gibson B, Nelson EA, Prescott RJ. Randomised, double blind placebo controlled trial of pentoxifylline in the treatment of venous leg ulcers. BMJ. 1999;319(7214): 875-878.

43. Falanga V, Fujitani RM, Diaz C, et al. Systemic treatment of venous leg ulcers with high doses of pentoxifylline: efficacy in a randomized, placebo-controlled trial. Wound Repair Regen. 1999;7(4): 208-213.

44. Belcaro G, Cesarone MR, Nicolaides AN, De Sanctis MT, Incandela L, Geroulakos G. Treatment of venous ulcers with pentoxifylline: a 6-month randomized, double-blind, placebo controlled trial. Angiology. 2002;53 Suppl 1:S45-S47.

45. Nikolovska S, Pavlova L, Petrova N, Gocev G, Ivanovski M. Pentoxifylline - efficient in the treatment of venous ulcers in the absence of compression? Acta Dermatovenerol Croat. 2002;10(1):9-13.

46. Jones JE, Nelson EA. Skin grafting for venous leg ulcers. Cochrane Database Syst Rev. 2007;2:CD001737.

47. Poskitt KR, James AH, Lloyd-Davies ER, Walton J, McCollum C. Pinch skin grafting or porcine dermis in venous ulcers: a randomised clinical trial. Br Med J (Clin Res Ed). 1987;294(6573):674-676.

48. Mol MA, Nanninga PB, van Eendenburg JP, Westerhof W, Mekkes JR, van Ginkel CJ. Grafting of venous leg ulcers. An intraindividual comparison between cultured skin equivalents and full-thickness skin punch grafts. J Am Acad Dermatol. 1991;24(1):77-82.

49. Teepe RG, Roseeuw DI, Hermans J, et al. Randomized trial comparing cryopreserved cultured epidermal allografts with hydrocolloid dressings in healing chronic venous ulcers. J Am Acad Dermatol. 1993;29(6):982-988.

50. Warburg FE, Danielsen L, Madsen SM, et al. Vein surgery with or without skin grafting versus conservative treatment for leg ulcers. A randomized prospective study. Acta Derm Venereol. 1994;74(4): 307-309.

51. Falanga V, Margolis D, Alvarez O, et al. Rapid healing of venous ulcers and lack of clinical rejection with an allogeneic cultured human skin equivalent. Human Skin Equivalent Investigators Group. Arch Dermatol. 1998;134(3):293-300.
52. Lindgren C, Marcusson JA, Toftgård R. Treatment of venous leg ulcers with cryopreserved cultured allogeneic keratinocytes: a prospective open controlled study. Br J Dermatol. 1998;139(2): 271-275.

53. Tausche AK, Skaria M, Böhlen L, et al. An autologous epidermal equivalent tissue-engineered from follicular outer root sheath keratinocytes is as effective as split-thickness skin autograft in recalcitrant vascular leg ulcers. Wound Repair Regen. 2003;11(4):248-252.

54. Krishnamoorthy L, Harding KG, Griffiths D, et al. The clinical and histological effects of Dermagraft in the healing of chronic venous leg ulcers. Phlebology. 2003;18(1):12-22.

55. Liu JY, Hafner J, Dragieva G, Seifert B, Burg G. Autologous cultured keratinocytes on porcine gelatin microbeads effectively heal chronic venous leg ulcers. Wound Repair Regen. 2004;12(2):148-156.

56. Navrátilová Z, Slonková V, Semrádová V, Adler J. Cryopreserved and lyophilized cultured epidermal allografts in the treatment of leg ulcers: a pilot study. J Eur Acad Dermatol Venereol. 2004;18(2): 173-179.

57. Omar AA, Mavor AI, Jones AM, Homer-Vanniasinkam S. Treatment of venous leg ulcers with Dermagraft. Eur J Vasc Endovasc Surg. 2004;27(6):666-672.

58. Howard DP, Howard A, Kothari A, Wales L, Guest M, Davies AH. The role of superficial venous surgery in the management of venous ulcers: a systematic review. Eur J Vasc Endovasc Surg. 2008;36(4): 458-465.

59. Guest M, Smith JJ, Tripuraneni G, et al. Randomized clinical trial of varicose vein surgery with compression versus compression alone for the treatment of venous ulceration. Phlebology. 2003;18(3): 130-136.

60. Zamboni P, Cisno C, Marchetti F, et al. Minimally invasive surgical management of primary venous ulcers vs. compression treatment: a randomized clinical trial. Eur J Vasc Endovasc Surg. 2003;25(4):313-318

61. van Gent WB, Hop WC, van Praag MC, Mackaay AJ, de Boer EM, Wittens $\mathrm{CH}$. Conservative versus surgical treatment of venous leg ulcers: a prospective, randomized, multicenter trial. J Vasc Surg. 2006;44(3):563-571.

62. Gohel MS, Barwell JR, Taylor M, et al. Long term results of compression therapy alone versus compression plus surgery in chronic venous ulceration (ESCHAR): randomised controlled trial. BMJ. 2007;335(7610):83.

63. Viarengo LM, Potério-Filho J, Potério GM, Menezes FH, Meirelles GV. Endovenous laser treatment for varicose veins in patients with active ulcers: measurement of intravenous and perivenous temperatures during the procedure. Dermatol Surg. 2007;33(10):1234-1242; discussion 1241-1242.

64. Yang D, Morrison BD, Vandongen YK, Singh A, Stacey MC. Malignancy in chronic leg ulcers. Med J Aust. 1996;164(12):718-720.
Clinical, Cosmetic and Investigational Dermatology

\section{Publish your work in this journal}

Clinical, Cosmetic and Investigational Dermatology is an international, peer-reviewed, open access, online journal that focuses on the latest clinical and experimental research in all aspects of skin disease and cosmetic interventions. All areas of dermatology will be covered; contributions will be welcomed from all clinicians and

\section{Dovepress}

basic science researchers globally. This journal is indexed on CAS The manuscript management system is completely online and includes a very quick and fair peer-review system, which is all easy to use. Visit http://www.dovepress.com/testimonials.php to read real quotes from published authors. 